\title{
TEMPO-oxidized $O$-acetyl galactoglucomannan oligomers: isolation and comprehensive structural elucidation
}

\author{
T. Eichinger ${ }^{1} \cdot$ J. Rahkila ${ }^{2} \cdot$ S. Willför ${ }^{1} \cdot$ C. Xu ${ }^{1}$
}

Received: 20 December 2017 / Published online: 23 November 2018

(c) The Author(s) 2018

\begin{abstract}
Hemicelluloses, the second most abundant polysaccharides right after cellulose, represent complex biopolymers whose structural features are not yet fully understood. Hence, the applicability of functionalized hemicellulose derivatives is still limited. Thus, the development of practical methods to reveal their structure is essential. Anionic, carboxylated GGM was prepared as the substrate, and their corresponding oligomers have been isolated and fully characterized for the first time in the present work. In order to accomplish this, each step has been optimized sequentially. Oxidized GGM was enzymatically digested, and the corresponding oligomers were isolated by preparative GPC. A toolset of wet chemistry and instrumental methods was used to characterize the isolated compounds in detail. Moreover, quantitative ${ }^{13} \mathrm{C}-\mathrm{NMR}$ combined with MALDI was demonstrated to deliver accurate analytical data compared to standard but time-consuming wet chemistry methods. The developed toolset is expected to be applicable to other plant polysaccharides and their derivatives as well.
\end{abstract}

$\begin{array}{ll}\text { Abbreviations } \\ \text { GGM } & O \text {-acetyl galactoglucomannan } \\ \text { GPC } & \text { Gel permeation chromatography } \\ \text { DP } & \text { Degree of polymerization } \\ \text { DS } & \text { Degree of substitution } \\ \text { HMDS } & \text { Hexamethyldisilazane } \\ \text { TEMPO } & 2,2,6,6 \text {-tetramethyl-1-piperidinyloxy }\end{array}$

Electronic supplementary material The online version of this article (https://doi.org/10.1007/s0022 6-018-1068-0) contains supplementary material, which is available to authorized users.

T. Eichinger

t.eichinger08@gmail.com

1 Johan Gadolin Process Chemistry Centre, c/o Laboratory of Wood and Paper Chemistry, Åbo Akademi University, Porthansgatan 3, 20500 Turku, Finland

2 Johan Gadolin Process Chemistry Centre, c/o Laboratory of Organic Chemistry, Åbo Akademi University, Biskopsgatan 8, 20500 Turku, Finland 


\section{TMCS Chlorotrimethylsilane \\ TMP Thermomechanical pulp \\ $M_{\mathrm{w}} \quad$ Weight average molar mass}

\section{Introduction}

In order to enhance the sustainability of lignocellulosic biorefinery concepts, new approaches for utilizing side streams must be found. The hemicellulose and lignin fraction represent two compound classes that still remain underrepresented with regard to their degree of utilization as a raw material. During the last couple of decades, significant research effort has been undertaken in order to extract water-soluble GGM from Norway spruce (Picea abies). Large quantities are available as a side product of papermaking as it can be recovered from the process water or thermomechanical pulp (Willför et al. 2003a) or directly from softwood by pressurized hot water extraction (Leppänen et al. 2011; Song et al. 2008). This compound family corresponding to a monomeric sugar ratio of 3.5-4.5:1:0.5-1 (Man/Glc/Gal) (Hannuksela and Du Penhoat 2004; Willför et al. 2003b, 2005) consists of a linear backbone of randomly distributed $(1 \rightarrow 4)$-linked $\beta$-D-mannopyranosyl and $(1 \rightarrow 4)$-linked $\beta$-D-glucopyranosyl units, with $(1 \rightarrow 6)$-linked $\alpha$-D-galactopyranosyl units coupled to the mannosyl units as shown in Fig. 1 (Hannuksela and Du Penhoat 2004; Willför et al. 2003b).

Typically, an average $M_{\mathrm{w}}$ of up to $20 \mathrm{~kg} / \mathrm{mol}$ corresponding to a DP of approximately 120 can be expected, of course depending on the isolation methods used (Hannuksela and Du Penhoat 2004; Willför et al. 2008).

The introduction of carboxyl groups at $\mathrm{C}_{6}$ position of GGM by TEMPO oxidation or just on $\alpha$-D-galactopyranosyl units by selective chemo-enzymatic approaches opens a new pathway toward anionic, carboxylated GGM (Parikka et al. 2012). Controlling the DP of GGM in the oligomeric region is giving access toward extensively

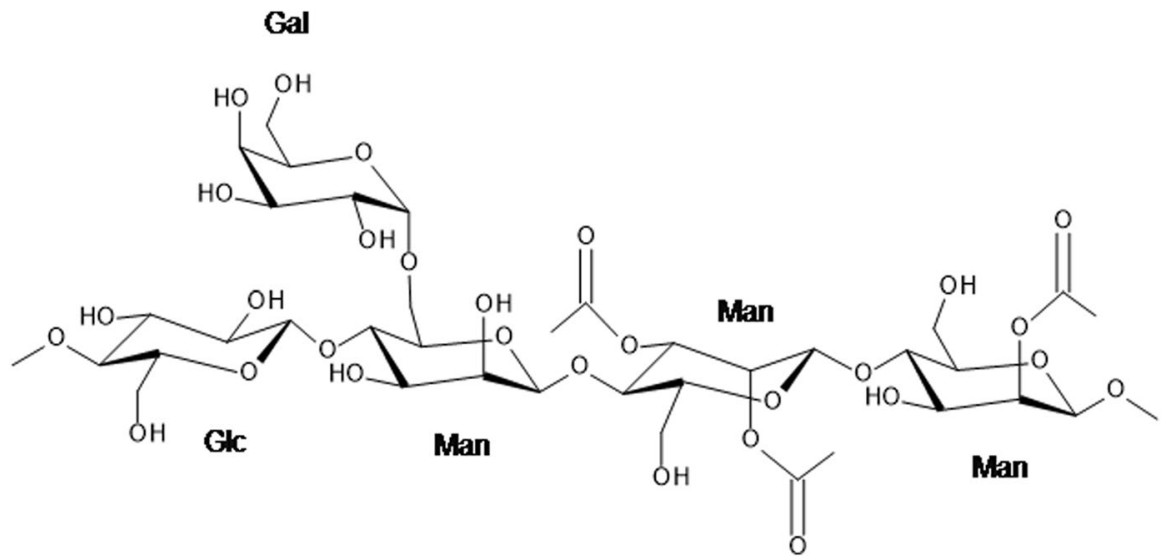

Fig. 1 Structure of GGM; monomeric units highlighted 
new properties, which are located between the monomer and the corresponding polymer (Zweckmair et al. 2016).

Adsorption of anionic, carboxylated low DP GGM to cellulose appears to be the method of choice to numerous high-value products such as specialty paper grades or diagnostic strips. An important advantage of this approach is that cellulose as such still remains unmodified leaving important material properties unchanged (Willför et al. 2008). However, GGM is not yet utilized on an industrial scale.

In the present work, D-raffinose was used as a model compound in order to optimize the preparation of TEMPO-oxidized low DP oligosaccharides. Consequently, a toolset was used to isolate anionic, carboxylated GGM oligomers: TEMPO oxidation was combined with enzymatic digestion followed by preparative GPC for the first time. In light of utilizing anionic, carboxylated low DP GGM in sophisticated applications, precise knowledge of the polymer structure including its substitution pattern becomes progressively important. Comprehensive NMR-spectroscopic methodology appears to be the method of choice for determining exact structural features of GGM as long as the polymer complexity is not exceeding a certain threshold (Capek et al. 2002; Ekholm et al. 2012; Hannuksela and Du Penhoat 2004; Lundqvist et al. 2002; Willför et al. 2003b). Even quantitative information is accessible when appropriate pulse techniques are used although the overall sensitivity might be rather low compared to mass spectrometry-based approaches (Jacobs and Dahlman 2001).

Gas chromatography-based approaches such as acid methanolysis or methylation analysis are capable of reporting the monomeric composition of complex samples, as well as the actual linkage of each monomeric compound at reasonable sensitivity (Willför et al. 2003b). Nevertheless, precise information on the substitution pattern remains inaccessible, since, for example, standard methylation analysis protocols apply conditions that may be detrimental to, for example, the $O$-acetyl groups present on the polymer (Willför et al. 2003b). Herein, a toolset is presented for the convenient qualitative elucidation of anionic, carboxylated low DP GGM.

\section{Materials and methods}

\section{Chemicals}

TEMPO 98\%, sodium borohydride 98 atom\%, hexamethyldisilazane, chlorotrimethylsilane, methanol $99.9 \%$, Dowex $50 \mathrm{~W} \times 8$ resin, sodium acetate $99 \%$, acetic anhydride $99 \%$ and LC-MS-grade ammonium hydroxide $25 \%$ were obtained from Sigma-Aldrich (Sigma-Aldrich Oy, Helsinki, Finland). Sodium bromine 99\%, ammonia $25 \%$, acetic acid $99.7 \%$, sodium hydroxide $99 \%$, sodium nitrate $99 \%$ and LC-MS-grade acetonitrile were purchased from Merck (Merck Oy, Espoo, Finland). Pyridine, sodium hypochlorite $18 \%$, and potassium dihydrogenphosphate $99.9 \%$ were provided by VWR (VWR International Oy, Helsinki, Finland). Hydrochloric acid was supplied by J.T. Baker (Avantor Performance Materials, Center Valley, PA, USA), while technical grade ethanol was purchased from Altia Oyj (Rajamäki, Finland). 


\section{Sample material preparation}

\section{GGM preparation}

GGM was obtained from Norway spruce (Picea abies) thermomechanical pulp (TMP) in the laboratory scale (Willför et al. 2003a). In brief, colloidal wood resin and aromatic residues were removed from TMP mill water in a preliminary purification step applying a cationic coagulant (Raifix 120, Raisio Chemicals Oy, Finland) and XAD-7 resin (Amberlite, Rohm and Haas, UK). GGM was concentrated by ultrafiltration and precipitated from ethanol followed by lyophilization.

\section{TEMPO oxidation}

Hundred milligrams of polysaccharide was dissolved in $40 \mathrm{~mL}$ of water (solution A); $152 \mathrm{mg}$ of sodium bromine and $1.2 \mathrm{mg}$ of TEMPO were dissolved in $10 \mathrm{~mL}$ of water (solution B). After cooling both solutions to $5{ }^{\circ} \mathrm{C}$, solution B was added to A followed by the dropwise addition of $2 \mathrm{~mL}$ sodium hypochlorite under continuous stirring. During the oxidation process, the $\mathrm{pH}$ was adjusted to $10-10.5$, while the reaction was cooled to $5{ }^{\circ} \mathrm{C}$ in an ice bath. The reaction was stopped by adjusting the $\mathrm{pH}$ to neutral followed by precipitation from ethanol, centrifugation, and lyophilization.

\section{Sodium borohydride reduction}

Hundred milligrams of TEMPO-oxidized GGM was dissolved in $40 \mathrm{~mL}$ of water. One milliliter of $2 \mathrm{M}$ ammonium hydroxide was added to stabilize $\mathrm{pH}$. Sixty milligrams of solid sodium borohydride was added for starting the reaction. Reduction was carried out for $2 \mathrm{~h}$ at room temperature under magnetic stirring. Excess of sodium borohydride was destroyed by the addition of $30 \%(\mathrm{v} / \mathrm{v})$ acetic acid. Solid GGM was recovered upon precipitation from ethanol, centrifugation, and subsequent lyophilization.

\section{Enzymatic hydrolysis}

Two hundred milligrams of polysaccharide was dissolved in $40 \mathrm{~mL} 5 \mathrm{mM}$ sodium acetate buffer at $\mathrm{pH}$ 6.0. After the addition of $100 \mu \mathrm{L}$ family GH5 endo-1,4- $\beta$ mannanase $300 \mathrm{U} / \mathrm{mL}$ (EC 3.2.78, source: Aspergillus niger; Megazyme, Wicklow, Ireland) and $50 \mathrm{mg}$ of cellulase (EC 232.734.4, source: Trichoderma reesei ATCC 26921; Sigma-Aldrich Oy, Helsinki, Finland), hydrolysis was allowed to proceed at $40{ }^{\circ} \mathrm{C}$ overnight. Subsequently, $\mathrm{pH}$ was adjusted to neutral followed by boiling for $10 \mathrm{~min}$ to deactivate the enzymes. 


\section{Trimethyl D-raffinose triuronate}

D-raffinose-pentahydrate (514 mg, $0.86 \mathrm{mmol}$; compound (A), c.f. Supplementary Material and Table S1) was subjected to TEMPO oxidation for $10 \mathrm{~min}$ at $5{ }^{\circ} \mathrm{C}$ as described above. Upon precipitation from acetone/water [1:1 (v/v)], $433.9 \mathrm{mg}$ of crude white solid product could be recovered (compound B).

Subsequently, esterification was carried out in analogy to Nudelman et al. (1998). In brief, $200.0 \mathrm{mg}$ of compound (B) was treated by freshly regenerated Dowex $50 \mathrm{~W} \times 8$ resin for transformation to the free acid.

Regeneration of the resin was carried out by stirring in diluted $\mathrm{HCl}$ followed by decantation. In a first step, the resin was stirred in $0.5 \mathrm{M} \mathrm{HCl}$ for 10 min followed by decantation. This procedure was repeated two additional times. Subsequently, the stirring/decantation procedure was carried out three times with $1 \mathrm{M}$ and $2 \mathrm{M} \mathrm{HCl}$ each. Finally, the resin was filtered on a glass filter followed by washing with deionized water. Afterward, the resin was ready for use. Small resin columns were packed and washed with deionized water until neutral $\mathrm{pH}$. After loading the sample, elution was carried out with approximately ten column volumes of water. The $\mathrm{pH}$ of the obtained product was monitored at around one $\mathrm{pH}$ unit below water, which was used for elution.

The product fraction was concentrated on the rotary evaporator until leaving only a small amount of water behind. Then, methanol was added followed by evaporation. Methanol addition and evaporation were additionally repeated four times.

Subsequently, anhydrous methanol was added and removed by evaporation. Finally, anhydrous methanol (40 mL, $988 \mathrm{mmol}), 2$,2-dimethoxypropane $(4.0 \mathrm{~mL}$, $32.6 \mathrm{mmol}$ ), and $400 \mu \mathrm{L}$ of $2 \mathrm{M}$ acetyl chloride in anhydrous methanol were added. Esterification under continuous stirring was carried out for 1 day at room temperature.

Subsequently, the solvent was removed by evaporation at 100 mbar. Redissolution in methanol followed by evaporation at 100 mbar was repeated three times. Finally, the evaporation was carried out at 10 mbar. $175.6 \mathrm{mg}$ of crude product was obtained appearing as a colorless, viscous liquid. Subsequently, purification was carried out by two chromatographic steps: At first, flash chromatography [ethyl acetate/ methanol 3:1 (v/v)] was applied. Subsequently, reversed-phase chromatography [water/acetonitrile 95:5 (v/v)] was used to isolate raffinose triuronate methyl ester (compound C, c.f. Supplementary Material and Table S2) as a colorless, viscous liquid (14.9 mg which corresponds to a yield of $6.4 \%$ relative to raffinose starting material).

In addition, $51.5 \mathrm{mg}$ of a reaction byproduct (compound D, c.f. Supplementary Material and Table S3) was isolated corresponding to a yield of $33.8 \%$ relative to raffinose starting material.

\section{O-methoxime D-raffinose triuronate}

Compound (B) obtained from TEMPO oxidation of raffinose was converted to the corresponding $O$-methoxime derivative (compound E). In brief, $4 \mathrm{mg}$ of compound (B) was dissolved in $0.8 \mathrm{~mL}$ of anhydrous pyridine and reacted with $15 \mathrm{mg}$ 
of $O$-methoxyamine hydrochloride at $70{ }^{\circ} \mathrm{C}$ for $1 \mathrm{~h}$. Subsequently, the solvent was removed under a stream of nitrogen. The product was dissolved in acetonitrile/water $(1: 1, \mathrm{v} / \mathrm{v})$, diluted and analyzed by ESI-MS profiling in negative ion mode using $0.1 \% \mathrm{NH}_{4} \mathrm{OH}$ as an eluent additive.

\section{Instrumental setup}

\section{Analysis of sugar composition}

The monomeric constituents of GGM were analyzed by acid methanolysis using $2 \mathrm{M} \mathrm{HCl}$ in methanol as a reagent at $100{ }^{\circ} \mathrm{C}$ for $3 \mathrm{~h}$. Subsequently, the monomers obtained were silylated and analyzed by GC (Sundberg et al. 1996; Willför et al. 2009).

\section{Acetylation analysis}

Ten milligrams of polysaccharide was dissolved in $9 \mathrm{~mL}$ water. The $\mathrm{pH}$ was adjusted to 11 by $1 \mathrm{M}$ sodium hydroxide solution prior to saponification at $70{ }^{\circ} \mathrm{C}$ for $2 \mathrm{~h}$ under continuous magnetic stirring. After cooling down the solution, the $\mathrm{pH}$ was adjusted to neutral. An appropriate amount of water was added to fill up the total sample volume to $10 \mathrm{~mL}$. Right before measurement, the $\mathrm{pH}$ was adjusted to 2.5 using $1 \mathrm{M}$ hydrochloric acid. HPLC analysis was carried out on an Agilent Infinity 1260 LC system (Agilent Technologies, Santa Clara, CA, USA). Separation was achieved on a Synergi $4 \mathrm{u}$ Hydro RP column $(4.6 \times 250 \mathrm{~mm}$ Phenomenex Inc., Torrance, CA, USA) connected to a pre-column. $20 \mathrm{mM}$ potassium dihydrogenphosphate $\mathrm{pH} 2.5,0.2 \mu \mathrm{m}$ filtered was used as the mobile phase at $1 \mathrm{~mL} / \mathrm{min}$ flow. Aliquots of $20 \mu \mathrm{L}$ were injected while monitoring UV at $210 \mathrm{~nm}$, as well as refractive index detection. Agilent ChemStation v.B04.03. (Agilent Technologies, Santa Clara, CA, USA) was used for operating the instrument, as well as for data analysis.

\section{Analysis of molar mass}

Average molar mass was determined by size exclusion chromatography coupled to a refractive index detector (RID 10A, Shimadzu Corporation, Japan). Two columns were connected in series: Ultrahydrogel 125 coupled to a Ultrahydrogel 500 column, (both $7.8 \times 300$ column, Waters Corp., Milford, MA, USA). 0.1 M sodium nitrate solvent was used at $0.5 \mathrm{~mL} / \mathrm{min}$ flow. The samples were filtered through a $0.22-\mu \mathrm{m}$ nylon syringe filter prior to injecting a $100 \mu \mathrm{L}$ aliquot. Calibration was based on pullulan standards using the following mass intervals (Da): 342, 1080, 5900, 9600, $21,100,47,100,107,000,200,000,344,000$, and 708,000. Data analysis was carried out using an in-house MATLAB script (MATLAB 2013b, MathWorks GmbH, Ismaning, Germany). 


\section{Nuclear magnetic resonance (NMR)}

NMR spectra were recorded on Bruker Avance III spectrometers operating at either $600.16 \mathrm{MHz}$ or $500.20 \mathrm{MHz}\left({ }^{1} \mathrm{H}\right)$ and $150.91 \mathrm{MHz}$ or $125.78 \mathrm{MHz}\left({ }^{13} \mathrm{C}\right)$. The $600 \mathrm{MHz}$ instrument was equipped with a Prodigy TCI inverted CryoProbe, and the $500 \mathrm{MHz}$ instrument was equipped with a Prodigy BBO CryoProbe. The complete assignment of proton and carbon spectra of the model compounds was carried out by using a standard set of NMR experiments: ${ }^{1} \mathrm{H}$ NMR, ${ }^{13} \mathrm{C}$ NMR, DQF-COSY, HSQC, and HMBC. The complexities of the ${ }^{1} \mathrm{H}$ spectra were reduced to monosaccharide level by using 1D-TOCSY. The chemical shifts are expressed on the $\delta(\mathrm{ppm})$ scale and are referenced to DMSO $\left(\delta_{\mathrm{H}}=2.17 \mathrm{ppm}, \delta_{\mathrm{C}}=39.39 \mathrm{ppm}\right)$. The chemical shifts are reported with two decimals in ${ }^{1} \mathrm{H}$ spectra and one decimal in ${ }^{13} \mathrm{C}$ spectra. In cases where this is not enough to distinguish two signals, an additional decimal is given. Coupling constants are reported with one decimal and only mentioned the first time they appear in order to avoid unnecessary bloating of the data. Accurate chemical shifts and coupling constants were obtained by simulating the spectra using the spectral simulating software PERCH.

\section{MALDI}

Acetylation of the sample material was carried out prior to MALDI analysis. Two milligrams of sample material was dissolved in $400 \mu \mathrm{L}$ pyridine. Upon adding $300 \mu \mathrm{L}$ of acetic anhydride, acetylation was carried out at $50{ }^{\circ} \mathrm{C}$ overnight with occasional shaking. After cooling to room temperature, the liquid phase was removed in a stream of nitrogen followed by lyophilization.

MALDI-TOF MS (Autoflex Speed, Bruker Daltonics, Bremen, Germany) equipped with a $1000 \mathrm{~Hz}$ SmartBeamTM-II laser was employed in this study.

Twenty milligrams/milliliters of 2,5-dihydroxybenzoic acid was dissolved in acetone containing $1 \mu \mathrm{L} / \mathrm{mL}$ trifluoroacetic acid (matrix); $0.5 \mathrm{mg}$ of sample was dissolved per $\mathrm{mL}$ acetone (sample). The matrix and the sample were mixed 1:1 (v/v) in an extra vial. After spotting of $1 \mu \mathrm{L}$ sample/matrix mixture on a MTP 384 Massive Target $\mathrm{T}$ plate (Bruker Daltonik, Bremen, Germany) following the dried droplet method, the samples were analyzed directly.

The instrument was controlled by FlexControl software (version 3.4.135.0) and was calibrated by commercial peptide standards (Bruker Daltonics, Bremen, Germany). MS spectra were acquired at $70 \%$ laser power under the linear positive ion mode. The detector voltage was set to $2850 \mathrm{~V}$, while 2000 shots from different sample spots were summarized. MS spectra were processed with the manufacturer's software (FlexAnalysis 3.3.80) using the SNAP algorithm with a signal/noise threshold of 6. MS spectra were exported into ASCII files for further processing using \pm 100 ppm extraction width.

\section{Preparative GPC of GGM oligomers followed by Fischer esterification}

The preparative HPLC system consisted of two high-pressure gradient Shimadzu LC-8A pumps with a $150 \mathrm{~mL} / \mathrm{min}$ pump head, a Shimadzu preparative $14 \mathrm{~mL}$ 
mixing chamber, Shimadzu SIL 10AP autosampler, Shimadzu RID 10A refractive index detector, and a Shimadzu SCL 10A system controller. Shimadzu Class VP software v. 6.12SP5 was used for operating the system and for data acquisition.

A $50 \times 800 \mathrm{~mm}$ column packed with BioGel P2 Gel fine grade (Bio-Rad, Valimotie, Finland) was used at $5.0 \mathrm{~mL} / \mathrm{min}$ isocratic $0.1 \mathrm{M}$ acetic acid flow. Fractions were collected automatically based on pre-determined retention time. After chromatographic separation, the collected fractions were evaporated to dryness yielding an amorphous solid.

Subsequently, esterification was carried out prior to NMR analysis in analogy to the raffinose model compound. The product obtained appeared as a colorless, viscous liquid and was directly dissolved in NMR solvent for analysis.

\section{Results and discussion}

\section{TEMPO oxidation of raffinose as a model compound}

As an initial step, raffinose was selected as a model compound to study TEMPO oxidation in more detail. ESI-MS profiling was used to tackle the formation of the desired triuronate including byproducts. After $10 \mathrm{~min}$ of oxidation time at $5{ }^{\circ} \mathrm{C}$, the corresponding raffinose triuronate was formed with a yield of approximately $40 \%$ relative to raffinose starting material (c.f. sample material preparation section), while only a small amount of byproducts, such as polyaldehydes, were detected. Increasing or decreasing the oxidation time greatly affected the yield of oxidized raffinose moieties (data not shown). Hence, $10 \mathrm{~min}$ of oxidation time was selected for the preparative protocol.

Mild methyl esterification was used to derivatize the uronic acid functionalities prior to conducting structural elucidation by NMR. However, ESI-MS profiling provided clear evidence that compound (D), c.f. Table S1, corresponding to raffinose triuronate eliminating the fructose unit, is almost exclusively formed during the methyl esterification step. Such an elimination reaction might be caused by the $\beta$-glycosidic linkage being located in rather close proximity to the carboxylic acid functionality introduced on $\mathrm{C}_{1}$ of fructose (c.f. compound $\mathrm{C}$ in Table $\mathrm{S} 1$ ).

Apart from the formation of raffinose triuronate and the corresponding byproduct (compound D), still a detectable amount of aldehydes might be left in the starting material as TEMPO oxidation was only carried out on a short timescale. In order to trace residual aldehydes, labeling by methoxyamine was carried out followed by ESI-MS profiling. Clear evidence for the presence of a small amount of residual aldehydes was found. It was decided to forego isolation and characterization of the derivatized aldehydes since the compounds formed consist of a number of positional isomers and appear to be beyond the scope of this work. However, in order to eliminate possible interference of residual aldehydes, a standard $\mathrm{NaBH}_{4}$ reducing step was included in the final protocol when oxidizing GGM by the TEMPO approach. As a next step, TEMPO oxidation of GGM was investigated in more detail. 


\section{TEMPO oxidation of GGM and isolation of their corresponding oligomers}

In order to control the number of introduced uronic acid functionalities, GGM was TEMPO-oxidized for different durations. While oxidation for 5 min resulted in a DS (uronic acids) of 0.17 , reacting for 30 min yielded a DS (uronic acids) of 0.48 . The DP as well as the amount of acetyl groups was not changed significantly throughout the whole procedure: DS (acetyl groups): 0.24.

In a subsequent step, enzymatic hydrolysis was carried out using 5 and $30 \mathrm{~min}$ TEMPO-oxidized GGM as substrates. A significantly different molar mass distribution was determined by GPC evaluating both oligomer fractions (c.f. Table 1 and Fig. A2): 5-min TEMPO oxidation resulted in smaller GGM oligomers compared to 30-min oxidation time. This relation appears to be consistent with the molar mass of the remaining polymers (c.f. Table 1). Enzymatic depolymerization might be limited by higher-level discriminating effects such as the presence of oxidized species, which obviously appears to be more pronounced at extended reaction time. However, the introduction of uronic acid functionalities is neither reflected by the presence of uronic acid functionalities in the remaining polymer nor in the oligomers isolated (c.f. Fig. 2). Probably, the presence of traces of residual aldehydes that are still left intact after $\mathrm{NaBH}_{4}$-reduction might cause enzymatic hindrance (c.f. Fig. 3).

Finally, the corresponding oligomers were isolated by preparative GPC (c.f. Fig. A1, Supplementary Material). Interestingly, only rather small differences with regard to the monomeric composition of the oligomers isolated after 5- and 30-min TEMPO oxidation could be found (c.f. Table 1).

The remaining polymers were investigated with regard to their monomeric composition. The presence of GlcA, GalA, and ManA appears to be much more pronounced compared to the oligomers isolated. Still, a significant amount of unoxidized Gal and Man is left behind in the remaining polymer even when oxidation is carried out for $30 \mathrm{~min}$. Interestingly, a rather significant difference with regard to the monomeric composition could be found between the two remaining polymers isolated. A short oxidation timescale causes accumulation of Gal in the remaining polymer. Extended oxidation duration leads to a decrease in the latter value, while a corresponding increase in GalA was not observed in the isolated

Table 1 Monomeric composition of the fractions isolated upon TEMPO oxidation of GGM ( $n=2$, average standard deviation $10.4 \%$ ) and molecular weight distribution

\begin{tabular}{llcccccccc}
\hline $\begin{array}{l}\text { TEMPO } \\
\text { oxidation } \\
(\mathrm{min})\end{array}$ & & Glc & Gal & Man & GlcA & GalA & ManA & $M_{\mathrm{w}}(\mathrm{g} / \mathrm{mol})$ & PDI \\
\hline- & Starting material & 1.0 & 0.7 & 3.1 & 0.1 & 0.2 & n.d. & 41,677 & 3.1 \\
5 & Oligomer & 1.0 & 0.2 & 3.2 & 0.3 & 0.1 & 1.3 & 6397 & 0.5 \\
& Remaining polymer & 1.0 & 6.2 & 3.0 & 1.5 & 1.0 & 4.4 & 15,524 & 9.3 \\
30 & Oligomer & 1.0 & 0.1 & 0.3 & 0.1 & n.d. & 0.6 & 12,150 & 1.4 \\
& Remaining polymer & 1.0 & 3.0 & 2.7 & 0.8 & 1.1 & 4.2 & 12,416 & 5.4 \\
\hline
\end{tabular}




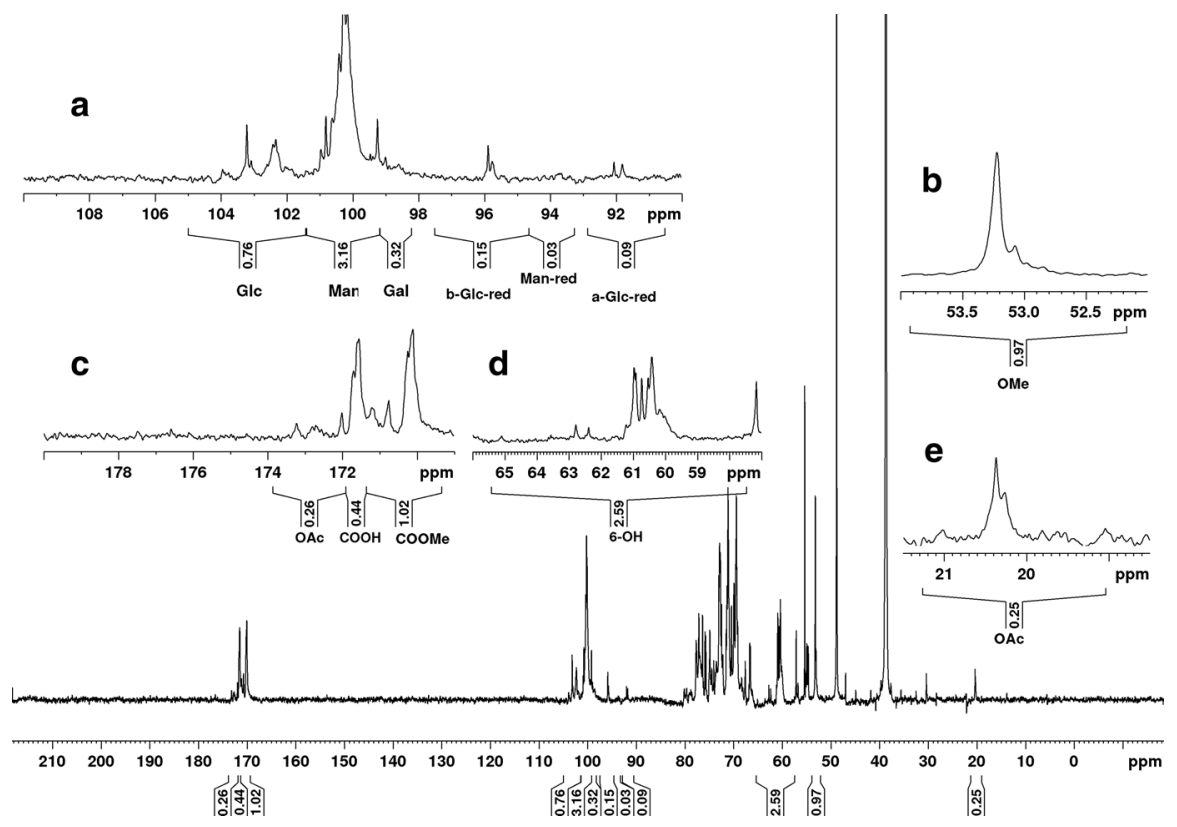

Fig. 2 Quantitative ${ }^{13} \mathrm{C}$ spectrum of anionic, carboxylated GGM upon methyl esterification

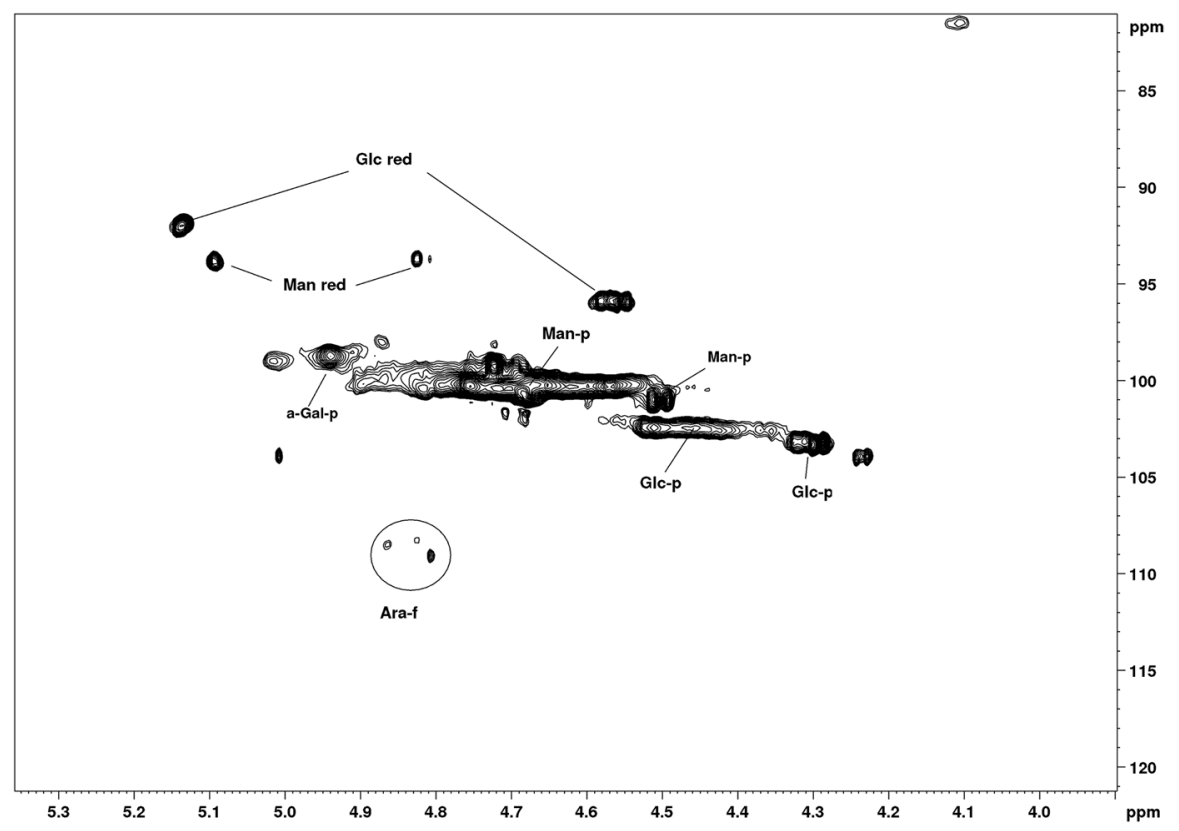

Fig. 3 HSQC spectrum of the anomeric signal range of anionic, carboxylated GGM upon methyl esterification 
oligomer. A quite similar phenomenon was observed in the case of GlcA and ManA as well (c.f. Table 1). However, the largest amount of oxidized moieties present on the isolated oligomer was found after $5 \mathrm{~min}$ of TEMPO oxidation.

Finally, 5-min TEMPO-oxidized GGM was selected as a substrate for preparative isolation of oligomers. The setup chosen enabled to isolate approximately $64 \mathrm{mg}$ oligomers/g starting material, which was characterized by comprehensive NMR techniques in a subsequent step.

\section{Characterization of anionic, carboxylated GGM oligomers by NMR}

The ${ }^{13} \mathrm{C}$-NMR signals were assigned based on literature data (Hannuksela and Du Penhoat 2004; Lundqvist et al. 2002) using the raffinose data set as a starting point. Quantitative ${ }^{13} \mathrm{C}$-NMR spectroscopy was used to determine the relative amount of structural features within the oligomer. The overall ratio of Man/Glc/ Gal monomer moieties was determined to be 3.2:1:0.46 based on the integration of the anomeric signals also taking the amount of $\mathrm{C}_{6}$-substituted $\mathrm{Gal}$ into account (c.f. Fig. 2, inset A). Acid methanolysis allowed to determine an overall ratio of 3.5:1:0.2, which is in rather good agreement with NMR.

A significant amount of reducing Glc could be detected with a ratio of Glc/ $\alpha$ Glc-red/ $\beta$-Glc-red of 1:0.19:0.11 (c.f. Fig. 2, inset A). An HSQC spectrum provides a more detailed view on the signal assignment in the anomeric range where traces of Ara seem to be present as an impurity (c.f. Fig. 3). In addition, a small amount of reducing Man was also detected (c.f. Fig. 2, inset A). Sodium borohydride reduction seems to leave a noticeable amount of reducing moieties with an unreasonable ratio behind although rather harsh reducing conditions were applied.

Anomeric signals for only $\alpha$-glycosidically linked galactose units were detected (c.f. Fig. 3). No corresponding signal for $\beta$-galactosides could be detected in the HSQC spectrum which is expected to appear below the Glc signals, i.e., 104-106 ppm of the ${ }^{13} \mathrm{C}$-axis and 4.4-4.8 ppm of the ${ }^{1} \mathrm{H}$-axis (c.f. Fig. 3).

The overall DS of uronic acids was determined to be 0.36 based on integrating the corresponding carbonyl- and free 6-OH signals (c.f. Fig. 2, insets C and D). However, in acid methanolysis followed by GC analysis, an overall DS of uronic acid of 0.27 was obtained. This deviation might be caused by integrating a slightly noisy signal from the ${ }^{13} \mathrm{C}$ spectrum on the one hand. On the other hand, a small but noticeable amount of uronic acids might not survive acid methanolysis causing an underestimation of the actual uronic acid content. However, a DS of uronic acid of at least 0.27 can safely be assumed.

In addition, an overall DS of methyl esterification of 0.69 was determined based on the integration of the carbonyl signals of the methyl-esterified and the free carboxylic acids (c.f. Fig. 2, inset C). Integrating the methyl peak (c.f. Fig. 2, inset B) instead of the carbonyl signal from the methyl ester gives very similar results. This number might encourage carrying out esterification under more harsh conditions or for an extended period of time. However, preliminary results esterifying carboxylated, anionic GGM oligomers with a drop of sulfuric acid or acidic methanol clearly 
showed that the latter variant is giving good methyl esterification yields while minimizing side reactions such as hydrolysis of glycosidic linkages, as observed in case of raffinose (data not shown). Further optimization of esterification time and temperature revealed that reacting 1 day at room temperature already gives a significant yield of methyl esters, which is rather hard to increase further.

Lactone formation appears as one possible reaction competing with esterification. However, a ring flip in order to transfer $\mathrm{C}_{6}$ into axial position would be required to form lactones. The formation of a five-membered lactone ring can safely be excluded due to the absence of a corresponding signal at 177-178 ppm in the carbon spectrum (c.f. Fig. 2, inset A). The formation of a six-membered lactone ring in case of 4-linked mannose appears to be very unlikely as well.

The overall DS of $O$-acetyl groups was determined to be 0.06 based on relating the integral of the acetyl carbonyl signal to the sum of $\mathrm{C}_{6}$-hydroxyls, carboxylic acids, and their methyl esters (c.f. Fig. 2, insets $C$ and D). Using the integral of the methyl signal from the $O$-acetyl group gives similar results. However, upon saponification of $O$-acetyl groups followed by HPLC analysis, a significantly higher overall DS of 0.24 was determined. The difference of the two results might be explained by quantifying the sum of the free acetic acid and the saponified $O$-acetyl groups by HPLC analysis. Hence, the DS of $O$-acetyl groups determined by NMR appears to be much more reliable.

The ratio of non-acetylated to acetylated Man was determined to be 14.6:1 based on NMR which appears to be significantly higher than 1.9:1 reported for GGM polymer (Willför et al. 2003b). The presence of $O$-acetyl groups might play an important role in enzymatic accessibility and water solubility. This might cause a significant difference between the DS of $O$-acetyl groups between the oligomer and the starting polymer.

Acetyl groups were shown to be attached to the $\mathrm{C}_{2}$ and $\mathrm{C}_{3}$ of the mannose units based on literature data (Van Hazendonk et al. 1996). The relative amount of 2-OAc to 3-O-Ac Man was approximated based on integrating the corresponding methyl signals in the ${ }^{13} \mathrm{C}$ spectrum (c.f. Fig. 2, inset E). However, as the two signals of interest are not very well resolved, the methyl signals from the corresponding ${ }^{1} \mathrm{H}$ spectrum were used to determine a more precise 2-O-Ac-to-3-O-Ac Man ratio of 1.5:1 (c.f. Fig. A3, inset A). The value appears to be slightly lower compared to the literature who reported a 2-O-Ac-to-3-O-Ac ratio of 2.2:1 considering isolated polymeric GGM (Willför et al. 2003b). However, O-Ac groups show a tendency to migrate under certain conditions rendering the 2-O-Ac-to-3-O-Ac ratio to equalize (Xu et al. 2010).

Upon structural elucidation by NMR, the isolated oligomers were qualitatively characterized by MALDI.

\section{Qualitative characterization of anionic, carboxylated GGM oligomers by MALDI}

In the first set of experiments, the GGM oligomer sample was analyzed in its native state using the methodology described in Enebro and Karlsson (2006). However, the oligomers could not be detected with reasonable signal intensity. The presence of an 


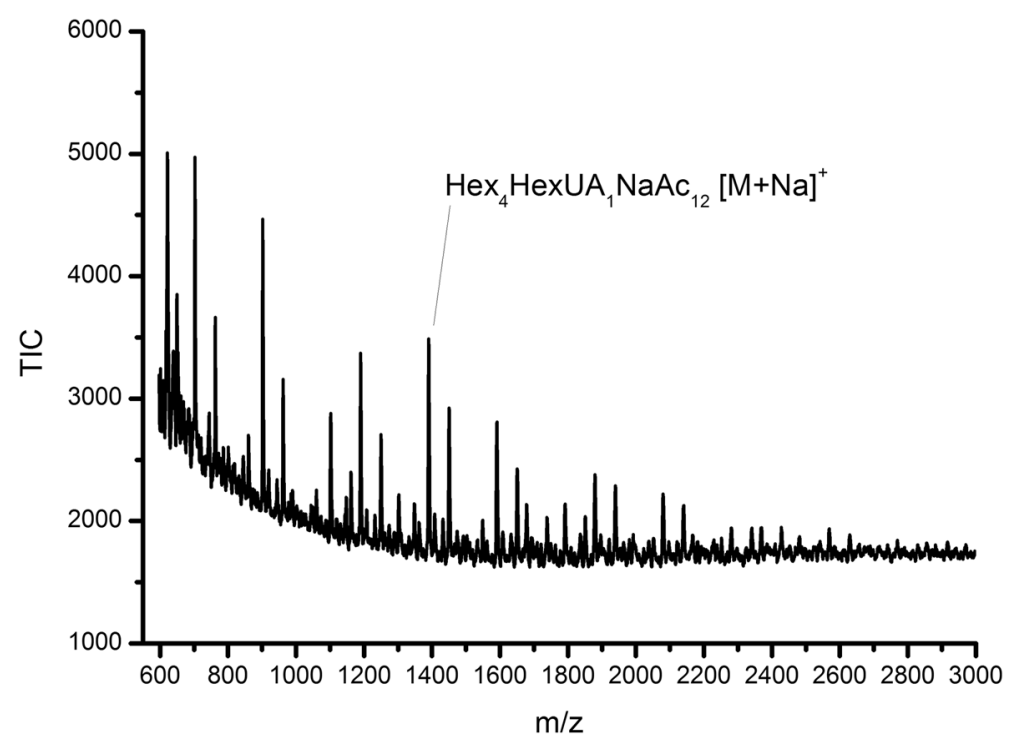

Fig. 4 MALDI analysis of TEMPO-oxidized GGM oligomers upon acetylation with one specific signal tentatively assigned

excessive amount of free hydroxyl groups, as well as a significant amount of uronic acid functionalities, might be a reasonable explanation. Hence, the sample material was acetylated prior to MALDI analysis and analyzed qualitatively using standard DHB matrix with reasonable signal intensity (c.f. Fig. 4).

Analyzing the sample material reflects the polydisperse nature of the isolated oligomers (c.f. Fig. 4). A significant increase in molar mass is expected upon acetylation, and MALDI only provides a rough approximation of the actual molar mass distribution. Individual signals appear as their singly or multiply sodiated compound in positive ion mode due to the presence of uronic acid functionalities (Chong et al. 2011). In Fig. 4, one representative signal was assigned to a tentative structural formula, which could be extended in analogy to the remaining signals. However, each tentative structure would need to be verified individually, which limits precise signal assignment a priori.

Comparing MALDI with GPC, data clearly show a significant difference: the molar mass distributions measured by MALDI appear to be significantly lower than those analyzed by GPC. Using pullulan standards for calibration, the presence of acetyl groups on the oligomer analyzed, as well as oligomer branching, might cause these measurement differences.

\section{Conclusion}

In the present work, a comprehensive toolset was established to isolate anionic, carboxylated GGM oligomers. GGM was TEMPO-oxidized at $5{ }^{\circ} \mathrm{C}$ on a minute timescale followed by enzymatic hydrolysis. Anionic, carboxylated GGM oligomers were successfully isolated in $\mathrm{mg}$ scale by preparative GPC. 
A number of analytical methodologies were evaluated regarding their usefulness to characterize anionic, carboxylated GGM oligomers. Methyl esterification was carried out as a sample preparation step prior to NMR analysis, which did not show any detrimental effect on the isolated material. Appropriate pulse techniques such as quantitative ${ }^{13} \mathrm{C}$-NMR appear to deliver accurate analytical data compared to standard wet chemistry methods.

Molar mass distribution of the isolated oligomers was approximated by MALDI and compared to standard GPC. The latter one appears to be significantly biased due to the usage of pullulan standards for calibration, oligomer branching, as well as the presence of acetyl groups. However, MALDI was shown to deliver more reliable molar mass data of TEMPO-oxidized GGM oligomers in this specific mass range.

In a subsequent investigation, oligomers, which ideally show a monodisperse molar mass distribution, would need to be isolated.

Acknowledgements Open access funding provided by Abo Akademi University (ABO). We would like to thank Mr. Lasse Tolonen for providing MATLAB scripts to evaluate GPC data. We express our gratitude to Prof. Thomas Rosenau and Prof. Antje Potthast for providing access to MALDI-TOF at BOKU Vienna. We also thank Dr. Sonja Schiehser for carrying out MALDI sample preparation. We are grateful to Prof. Maija Tenkanen from the University of Helsinki for numerous valuable discussions. This work was part of the activities at the Johan Gadolin Process Chemistry Centre appointed by Åbo Akademi University and of the Johan Gadolin Scholarship Program.

Open Access This article is distributed under the terms of the Creative Commons Attribution 4.0 International License (http://creativecommons.org/licenses/by/4.0/), which permits unrestricted use, distribution, and reproduction in any medium, provided you give appropriate credit to the original author(s) and the source, provide a link to the Creative Commons license, and indicate if changes were made.

\section{References}

Capek P, Alföldi J, Lišková D (2002) An acetylated galactoglucomannan from Picea abies L. Karst. Carbohydr Res 337:1033-1037. https://doi.org/10.1016/s0008-6215(02)00090-3

Chong S-L, Nissilä T, Ketola RA, Koutaniemi S, Derba-Maceluch M, Mellerowicz EJ, Tenkanen M, Tuomainen P (2011) Feasibility of using atmospheric pressure matrix-assisted laser desorption/ionization with ion trap mass spectrometry in the analysis of acetylated xylooligosaccharides derived from hardwoods and Arabidopsis thaliana. Anal Bioanal Chem 401:2995. https://doi.org/10.1007/ s00216-011-5370-z

Ekholm FS, Ardá A, Eklund P, André S, Gabius HJ, Jiménez-Barbero J, Leino R (2012) Studies related to Norway spruce galactoglucomannans: chemical synthesis, conformation analysis, NMR spectroscopic characterization, and molecular recognition of model compounds. Chem Eur J 18:1439214405. https://doi.org/10.1002/chem.201200510

Enebro J, Karlsson S (2006) Improved matrix-assisted laser desorption/ionisation time-of-flight mass spectrometry of carboxymethyl cellulose. Rapid Commun Mass Spectrom 20:3693-3698. https:// doi.org/10.1002/rcm.2786

Hannuksela T, Du Penhoat CH (2004) NMR structural determination of dissolved $O$-acetylated galactoglucomannan isolated from spruce thermomechanical pulp. Carbohydr Res 339:301-312. https:// doi.org/10.1016/j.carres.2003.10.025

Jacobs A, Dahlman O (2001) Enhancement of the quality of MALDI mass spectra of highly acidic oligosaccharides by using a Nafion-coated probe. Anal Chem 73:405-410. https://doi.org/10.1021/ac001 $222 \mathrm{i}$ 
Leppänen K, Spetz P, Pranovich A, Hartonen K, Kitunen V, Ilvesniemi H (2011) Pressurized hot water extraction of Norway spruce hemicelluloses using a flow-through system. Wood Sci Technol 45:223-236. https://doi.org/10.1007/s00226-010-0320-z

Lundqvist J, Teleman A, Junel L, Zacchi G, Dahlman O, Tjerneld F, Stålbrand H (2002) Isolation and characterization of galactoglucomannan from spruce (Picea abies). Carbohydr Polym 48:29-39. https://doi.org/10.1016/s0144-8617(01)00210-7

Nudelman A, Bechor Y, Falb E, Fischer B, Wexler BA, Nudelman A (1998) Acetyl chloride-methanol as a convenient reagent for: A) quantitative formation of amine hydrochlorides B) carboxylate ester formation C) mild removal of N-t-Boc-protective group. Synth Commun 28:471-474. https://doi. org/10.1080/00397919808005101

Parikka K, Leppänen AS, Xu C, Pitkänen L, Eronen P, Österberg M, Brumer H, Willför S, Tenkanen M (2012) Functional and anionic cellulose-interacting polymers by selective chemo-enzymatic carboxylation of galactose-containing polysaccharides. Biomacromolecules 13:2418-2428. https://doi. org/10.1021/bm300679a

Song T, Pranovich A, Sumerskiy I, Holmbom B (2008) Extraction of galactoglucomannan from spruce wood with pressurised hot water. Holzforschung 62:659-666. https://doi.org/10.1515/hf.2008.131

Sundberg A, Sundberg K, Lillandt C, Holmbom B (1996) Determination of hemicelluloses and pectins in wood and pulp fibres by acid methanolysis and gas chromatography. Nord Pulp Pap Res J 11:216219. https://doi.org/10.3183/NPPRJ-1996-11-04-p216-219

Van Hazendonk JM, Reinerik EJM, De Waard P, Van Dam JEG (1996) Structural analysis of acetylated hemicellulose polysaccharides from fibre flax (Linum usitatissimum L.). Carbohydr Res 291:141-154

Willför S, Rehn P, Sundberg A, Sundbgerg K, Holmbom B (2003a) Recovery of water-soluble acetylgalactoglucomannans from mechanical pulp of spruce. Tappi J 2:27-32

Willför S, Sjöholm R, Laine C, Roslund M, Hemming J, Holmbom B (2003b) Characterisation of watersoluble galactoglucomannans from Norway spruce wood and thermomechanical pulp. Carbohydr Polym 52:175-187. https://doi.org/10.1016/s0144-8617(02)00288-6

Willför S, Sundberg A, Hemming J, Holmbom B (2005) Polysaccharides in some industrially important softwood species. Wood Sci Technol 39:245-258. https://doi.org/10.1007/s00226-004-0280-2

Willför S, Sundberg K, Tenkanen M, Holmbom B (2008) Spruce-derived mannans: a potential raw material for hydrocolloids and novel advanced natural materials. Carbohydr Polym 72:197-210. https:// doi.org/10.1016/j.carbpol.2007.08.006

Willför S, Pranovich A, Tamminen T, Puls J, Laine C, Suurnäkki A, Saake B, Uotila K, Simolin H, Hemming J, Holmbom B (2009) Carbohydrate analysis of plant materials with uronic acid-containing polysaccharides: a comparison between different hydrolysis and subsequent chromatographic analytical techniques. Ind Crops Prod 29:571-580. https://doi.org/10.1016/j.indcrop.2008.11.003

Xu C, Leppänen AS, Eklund P, Holmlund P, Sjöholm R, Sundberg K, Willför S (2010) Acetylation and characterization of spruce (Picea abies) galactoglucomannans. Carbohydr Res 345:810-816. https:// doi.org/10.1016/j.carres.2010.01.007

Zweckmair T, Oberlerchner JT, Böhmdorfer S, Bacher M, Sauerland V, Rosenau T, Potthast A (2016) Preparation and analytical characterisation of pure fractions of cellooligosaccharides. J Chromatogr A 1431:47-54. https://doi.org/10.1016/j.chroma.2015.12.090

Publisher's Note Springer Nature remains neutral with regard to jurisdictional claims in published maps and institutional affiliations. 